\title{
Presencia y afectación del síndrome de burnout en instructores de TH-67 de la Escuela de Helicópteros para las Fuerzas Armadas de Colombia
}

| Fecha de recibido: 27 de abril del 2020 | Fecha de aprobación: 28 de septiembre del 2020 |

\section{Alexander Arboleda Medina \\ Magíster en Seguridad Operacional \\ Fuerza Aérea Colombiana \\ Rol de investigador: experimental y teórico https://orcid.org/0000-0001-5496-7223 \\ \lextramax@hotmail.com}

\section{Bryan Ramírez}

Magíster en Calidad y Gestión Integral

Docente de la Escuela de Postgrados de la Fuerza Aérea Colombiana (EPFAC)

Grupo de investigación CELSO

Rol de investigador: escritura

https://orcid.org/0000-0003-1562-5479

\bryan.ramirez@epfac.edu.co

\section{Leidy Gabriela Ariza Ariza}

Doctora en Educación Ambiental

Docente e investigadora de la Escuela de Postgrados de la Fuerza Aérea Colombiana (EPFAC)

Grupo de investigación CELSO

Rol de investigador: escritura

https://orcid.org/0000-0001-5970-3059

$\square$ leidygabrielaa@yahoo.es

\section{Nohora Rodríguez Guerrero}

Magíster en Bioética

Maestría en Epidemiología Clínica. Médica cirujana.

Docente de la Escuela de Postgrados de la Fuerza

Aérea Colombiana (EPFAC) y la Maestría en Seguridad Operacional (MAESO) Grupo de investigación CELSO

Rol de investigador: escritura https://orcid.org/0000-0002-2064-639X

$\triangle$ nirodrigu21@gmail.com

Cómo citar este artículo: Arboleda Medina, A., Ramírez, B., Ariza Ariza, L. G., \& Rodríguez Guerrero, N. (2020).

Presencia y afectación del síndrome de burnout en instrucciones de TH-67 de la Escuela de Helicópteros para las Fuerzas Armadas de Colombia. Revista Ciencia y Poder Aéreo, 15(2), 6-17. https://doi.org/10.18667/cienciaypoderaereo.679 


\section{Presencia y afectación del síndrome de burnout en instructores de TH-67 de la Escuela de Helicópteros para las Fuerzas Armadas de Colombia}

Resumen: la presente investigación pretende estimar la presencia del síndrome de burnout también conocido como "síndrome de desgaste profesional" o "síndrome del quemado en el trabajo", y su relación con la carga laboral administrativa y operativa en la población de pilotos instructores de TH-67 en la Escuela de Helicópteros para las Fuerzas Armadas de Colombia. Se realizó un estudio descriptivo transversal con enfoque mixto para identificar la presencia del síndrome de burnout mediante la aplicación del test de Maslach burnout inventory, el cual evalúa tres dimensiones: realización personal, agotamiento emocional y despersonalización. De igual forma, se tuvo en cuenta la asociación con las condiciones laborales de los pilotos instructores considerando tanto las operaciones de instrucción y vuelo, como las labores administrativas y militares. Los resultados del estudio mostraron que la presencia del síndrome de burnout en los pilotos instructores no es frecuente, como se verá en los resultados de las dimensiones analizadas. Así mismo, no se observó asociación entre los factores laborales (horas de preparación, tiempo en el cargo administrativo, horas en cargo adicional, carga administrativa y años de instructor en $\mathrm{TH}-67$ ).

Palabras clave: condiciones laborales; instructores; síndrome de burnout.

Abstract: This research seeks to estimate the incidence of burnout syndrome, also known as occupational burnout syndrome, and its relationship with the administrative and operational workload of TH-67 pilot instructors at the Helicopter School of the Colombian Armed Forces. A descriptive and cross-sectional study was carried out in order to identify the presence of burnout syndrome. The study involved the application of the Maslach Burnout Inventory, which evaluates three dimensions: personal accomplishment, emotional exhaustion, and depersonalization. In addition, the association with the working conditions of flight instructors in regard to training and flight operations as well as administrative and military tasks were also considered in this study. The results showed that burnout syndrome is not a frequent condition in pilot instructors, since emotional exhaustion and depersonalization dimensions reported low levels in most participants, while personal fulfillment recorded high levels. Besides, no association was observed between the studied labor factors (hours of training, time in an administrative position, hours in an additional position, administrative burden, and number of years as a TH-67 instructor).

Keywords: Working conditions; instructors; burnout syndrome.

Resumo: A pesquisa tem como objetivo estabelecer a presença do Síndrome de Burnout, também conhecido como Síndrome do Esgotamento Profissional e sua relação com a carga laboral administrativa e operativa na população de pilotos instrutores de TH-67 na Escola de Helicópteros das Forças Armadas da Colômbia. Para tal fim, foi feito um estudo descritivo transversal com uma abordagem misturada, para identificar a presença do Síndrome de Burnout a partir da aplicação do Test de Maslach Burnout Inventory, que avalia três dimensões; realização pessoal, esgotamento emocional e despersonalização. Além, da associação com as condições laborais dos pilotos instrutores, considerando tanto o treinamento e as operações de voo, quanto as tarefas administrativas e militares. Os resultados obtidos mostraram que a presença do Síndrome de Burnout nos pilotos instrutores não é frequente, dado que na dimensão de esgotamento emocional a maioria da população apresento baixo nível. No entanto, na dimensão de realização pessoal o nível foi alto, e na dimensão de despersonalização o nível foi baixo no pilotos instrutores avaliados. Da mesma forma, não foi observada a associação entre os fatores laborais (horas de preparação, tempo no cargo administrativo, horas no cargo adicional, carga administrativa e anos de instrução em TH-67).

Palavras-chave: Condições laborais; Instrutores; Síndrome de Burnout. 


\section{Introducción}

El presente trabajo tiene por propósito evaluar el factor humano como condicionante de la seguridad de los vuelos en el escuadrón de TH-67. Teniendo en cuenta que el sector aeronáutico es considerado un elemento determinante en el desarrollo económico y social de los países (Centro de Información de las Naciones Unidas, CINU), especialmente en la época globalizada del siglo xxı. En ese sentido, para un país como Colombia la aviación civil y la aviación militar constituye la defensa, control y soberanía del espacio aéreo en todo el territorio nacional como es la visión y misión de Fuerza Aérea Colombiana.

Según Rubio (2010), del 70 \% al 80 \% de los accidentes de aviación en el mundo están relacionados con errores o fallas humanas provenientes de los pilotos, la tripulación y demás personal encargado del mantenimiento y operación de las aeronaves. Por tal motivo, es importante estimar la presencia del síndrome de burnout, también conocido como "síndrome de desgaste profesional" o "síndrome del quemado en el trabajo" (SQT) y su relación con la carga laboral administrativa y operativa en la población de pilotos instructores de TH-67 en la Escuela de Helicópteros para las Fuerzas Armadas de Colombia, ya que las condiciones de salud física y mental de las tripulaciones son un tema prioritario en el ámbito mundial en cuanto a términos de seguridad aérea. La Escuela de Helicópteros de la Fuerza Pública tiene altos estándares de calidad en los diferentes niveles y procesos de formación que allí se desarrollan, forma pilotos militares de helicópteros $\mathrm{TH}-67$ de manera continua a lo largo del año, lo que implica un alto grado de exigencia y responsabilidad para el personal, tanto instructores como alumnos, y ubica al factor humano como decisivo para el logro de los objetivos en el proceso de instrucción y formación de nuevos pilotos.

A través de los años se han realizado estudios que indican el estado de salud y las condiciones laborales de las personas, como el caso del síndrome de burnout, que según Golembiewski, Munzenrider \& Carter (1983), a menudo puede afectar a muchas profesiones, entre ellas profesionales de la salud, policías, militares, profesores y estudiantes.

A este respecto, para Alonso (2009) tanto la salud física como la salud mental de las tripulaciones, "no solo de los pilotos", juega un papel importante en la seguridad de los vuelos civiles y militares. De allí radica la importancia de conocer el estado actual de los pilotos instructores de $\mathrm{TH}-67$, en términos de salud mental, estrés, fatiga y agotamiento, por lo cual, al realizar un estudio del síndrome de burnout en los instructores, permite conocer si están siendo afectados por las condiciones laborales.

\section{Marco referencial}

El síndrome de burnout se asocia directamente con el trabajo, afecta en especial a aquellas personas o profesionales que tienen contacto directo con otro personal a lo largo de la jornada laboral. Aranda (2006) (citado en Quiceno \& Vinaccia-Alpi, 2007) menciona que la palabra burnout es un término anglosajón que significa estar quemado en el trabajo, fue descrito en Estados Unidos por el psicólogo clínico Freudenberger en 1974, quien puntualizó sobre la existencia en los profesionales de la sensación de fracaso y agotamiento, resultado de una sobrecarga de esfuerzo laboral que afectaba tanto el estado físico como el mental de quienes lo padecían. Las personas utilizan recursos cognitivos y conductuales cuando se encuentran en situaciones adversas y estresantes, en este caso se le conoce como afrontamiento, e implica hacer un esfuerzo cognitivo, emocional y de conducta con el fin de contrarrestar factores estresores externos e internos (Montoya-Zuluaga \& Moreno-Moreno, 2012).

El burnout es un síndrome costoso para los individuos afectados y para las compañías en las que estos laboran, ya que puede causar síntomas como fatiga, falta de concentración, problemas de memoria y cambios de personalidad (desinterés, cinismo, agresión). A la vez, presentan otros signos físicos comunes que incluyen dolores de cabeza, trastornos gastrointestinales y problemas cardiovasculares, que se traducen 
en detrimento de la producción, productividad, y por ende, aumento de riesgos laborales (Rholetter, 2013). Dentro de los síntomas asociados al burnout, se caracterizan los factores cognitivos, conductuales, emocionales, físicos y defensivos, así (tabla 1):

Tabla 1.

Síntomas más frecuentes del síndrome de burnout

- Desaparición de expectativas, modificación de autoconcepto, desorientación cognitiva, distracción, cinismo, pérdida de la autoestima, del significado, de los valores y de la creatividad.

- Evitación de responsabilidad, ausentismo e intensión de abandonar las responsabilidades, desvalorización, autosabotaje, desvalorización del propio trabajo, desorganización, sobreimplicación, evitación de decisiones y aumento del consumo de cafeína, alcohol, tabaco y sustancias psicoactivas.

- Indefensión, sentimientos de fracaso, desesperanza, irritabilidad, desilusión, pesimismo, hostilidad, falta de tolerancia y supresión de síntomas.

- Cefaleas, síntomas osteomusculares especialmente dorsales, quejas psicosomáticas, pérdida del apetito, cambio del peso corporal, dificultades sexuales, problemas de sueño, fatiga crónica, enfermedades cardiovasculares y alteraciones gastrointestinales.

- Aislamiento, evitación de contacto profesional, conflictos interpersonales, malhumor y formación de grupos críticos.

- Fatiga crónica, dolores de cabeza frecuentes, problemas de sueño, úlceras u otros desordenes gastrointestinales, pérdida de peso, hipertensión, asma, dolores musculares en el cuello, y en las mujeres, pérdida de ciclos menstruales.

- Absentismo laboral, abuso de drogas, aumento de la conducta violenta, comportamiento de alto riesgo como la conducción suicida y juegos de azar peligrosos.

- Distanciamiento afectivo, irritabilidad, recelos, incapacidad para concentrarse, baja autoestima, deseos de abandonar el trabajo e ideas suicidas.

- Negación de las emociones, atención selectiva, ironía, racionalización y desplazamiento de afectos (Párraga, 2005).

Fuente: adaptado de Estrella-Silva \& Portalanza (2015).

Dado el impacto del síndrome de burnout, se han desarrollado investigaciones con pruebas generales y específicas que lo detectan y lo miden. Las generales, cuestionario breve de burnout (C.B.B) y Maslach Burnout Inventory-General Survey (MBI-GS); y las específicas, Cuestionario para la Evaluación del Síndrome de Quemarse por el Trabajo (CESQT), Holland Burnout
Assessment Survey Teacher Burnout Questionnaire, MBI-Educators Survey (MBI-ES), Maslach Burnout Inventory (MBI), Human Services Survey (MBI-HSS) y Staff Burnout Scale for Health Professionals (SBS-HP) (Quiceno \& Vinaccia-Alpi, 2007).

Para Patlán (2013), el síndrome de burnout es un estado de estrés o de cansancio asociado a las condiciones laborales de trabajadores en profesiones que interactúan con usuarios. Se destacan trabajadores de instituciones educativas y de salud, con algunas profesiones o cargos en mayor predisposición a sufrirlo, y la consecuencia de alteraciones emocionales, mentales y físicas en quienes lo padecen.

Las dimensiones que se evalúan para la identificación del burnout, en primer lugar, incluyen el agotamiento emocional, estado en el que el individuo presenta sentimientos de cansancio frente a las labores o exigencias del trabajo, y, por ende, no afronta adecuadamente las responsabilidades de su cargo. En segundo término, se evalúa la dimensión despersonalización, definida como el desarrollo de actitudes y sentimientos negativos y cínicos acerca de los clientes o usuarios, traducidos en una deshumanizada percepción de los demás, característica que se expresa en conductas de rechazo hacia los usuarios del servicio, a los cuales se les culpa de su situación actual, burocratizando la relación con ellos (Sierra-Siegert, 2008). La tercera gran dimensión involucrada en la valoración, es la falta de realización personal, evidenciada como la tendencia del trabajador a evaluarse negativamente a sí mismo y hacia el trabajo, la pérdida de interés, baja autoestima, aislamiento en la tarea, sensación de incompetencia personal, irritabilidad, descontento y reducida satisfacción con los logros laborales (Martínez et al., 2012).

El cuestionario Maslach Burnout Inventory (MBI), elaborado por Maslach y Jackson (1986), ha sido uno de los más utilizados para esta evaluación. Con el tiempo ha tenido variaciones, ajustes y diferentes modificaciones, pero cuenta con importantes ventajas, entre las que se encuentran la gran aceptación internacional, el apoyo empírico a la estructura factorial, la evidencia de validez concurrente y de validez divergente (Olivares-Faúndez \& Gil-Monte, 2009). 


\section{Metodología}

La metodología empleada tiene un enfoque mixto con alcance descriptivo transversal, la población de estudio está constituida por pilotos instructores de ala rotatoria de las Fuerzas Militares de Colombia, específicamente de TH-67 de la Escuela de Helicópteros de la Fuerza Pública. La muestra se enfocó en el mayor número de instructores que aceptaron participar en el estudio durante el último trimestre del 2017, teniendo en cuenta la disponibilidad de la Escuela y de los pilotos, y que coincidiera en la fase de vuelo en la que se encontraban al momento de la recolección de la información. De modo que, en total fueron 27 personas las que participaron del estudio.

Aunque la población es limitada, cabe destacar la importancia del estudio y el impacto que puede generar en dos aspectos: en lo económico, disminuye el alto costo de mantenimiento o reparación de las aeronaves; y en el factor humano, minimiza los riesgos de incidentes asociados a la falta de concentración, toma inapropiada de decisiones, cansancio y estrés, ya que son las vidas de los oficiales y suboficiales de la Fuerza Aérea las que se arriesgan en vuelos que no cumplen con los estándares de seguridad. Para el estudio se usó el criterio de transferencia, es decir, se toma un instrumento validado y contrastado por otros investigadores como es el test de Maslach Burnout Inventory.

En el antecedente registrado del grupo de seguridad de Comando Aéreo de combate $n .{ }^{\circ} 4$, se evidencia que el factor humano es determinante en los incidentes y accidentes aéreos en vuelos de instrucción en la Escuela de Helicópteros de la Fuerza Pública: el 25 \% de los eventos en el 2015 fueron por fallas de factor humano en vuelos de instrucción, los cuales aumentaron significativamente al $60 \%$ en el 2016 (FAC, 2016).

Así mismo, es importante señalar que en la actualidad la Escuela de Helicópteros no cuenta con estudios que evidencien el estado físico y mental de los pilotos instructores en términos de estrés, agotamiento $y$ fatiga, por lo que este estudio se convierte en un punto de partida al formular ¿Qué tan frecuente es el síndrome de burnout y su relación con la carga laboral administrativa y operativa en la población de pilotos instructores de TH-67 en la Escuela de Helicópteros para las Fuerzas Armadas de Colombia?

Como se ha mencionado, la investigación desarrolló un análisis descriptivo transversal de enfoque mixto, dando mayor prioridad al aspecto cualitativo, ya que lo que se pretendía era identificar la incidencia del síndrome en los pilotos instructores, sin embargo, el instrumento utilizado también brinda información cuantitativa. Además, la metodología mixta es más flexible para el investigador por su carácter sociocrítico y heurístico. En tal sentido, el objetivo de la investigación descriptiva era determinar las causas del problema de investigación, entendiéndose por descriptivo, lo que para Bernal (2010) es reseñar las características más significativas de las situaciones o fenómenos objeto de estudio, se trata de indagar en aspectos relacionados con el factor humano en la Escuela de Helicópteros de las Fuerzas Militares al determinar la prevalencia del síndrome de burnout, e identificar las características de las condiciones laborales de los instructores de TH-67. Así mismo, el estudio es transversal, en tanto que la recolección de datos se realiza en un solo momento mediante el test (Hernández-Sampieri, Fernández-Collado \& Baptista-Lucio, 2014).

El test de Maslach Burnout Inventory validado en español por Pando-Moreno, Aranda-Beltrán \& López-Palomar (2015), se constituye de 22 ítems definidos con las siguientes escalas valorativas: nunca (0); alguna vez al año o menos (1); una vez al mes o menos (2); algunas veces al mes (3); una vez a la semana (4); varias veces a la semana (5); y a diario (6); junto al cuestionario propuesto validado por pares, con 15 ítems para identificar y caracterizar las condiciones laborales actuales de los oficiales instructores en términos de tiempo, intensidad laboral, cargo, entre otros.

Los resultados obtenidos fueron tabulados utilizando un código de identificación alfanumérico para cada participante. Posteriormente, los datos se analizaron estadísticamente con medidas de tendencia central por medio del paquete de software estadístico Stata 13, para determinar la presencia del síndrome de burnout y los factores laborales más destacados del cuestionario. Por último, de forma exploratoria, se 
realizó un análisis bivariado utilizando la técnica del chi cuadrado para establecer la relación entre las horas de instructor y las cargas administrativas con las tres dimensiones del test.

\section{Resultados}

Como ya se ha dicho, los datos obtenidos sobre las dimensiones del Test de Maslach Burnout Inventory, analizados con Stata 13, dejaron los siguientes resultados: la dimensión agotamiento emocional (E.E) presenta un $57 \%$ con nivel bajo, seguido del nivel medio con $36 \%$ y con nivel bajo el $7 \%$ de la población. Por otro lado, en la dimensión de realización personal (P.A) se puede evidenciar que no existe población en el ítem bajo, y, por el contrario, la población se encuentra entre los parámetros medio y alto, siendo el $14 \%$ de nivel medio y el $86 \%$ de nivel alto, indicando que gran porcentaje de la población se encuentra satisfecho con el trabajo que realiza. Al analizar la dimensión de despersonalización (D), se encontró que el 93 \% de la población objeto de estudio en el nivel bajo, el restante $7 \%$ se encuentra en el nivel medio y no existe población en el nivel alto de despersonalización (figura 1).

Dimensiones del burnouten instructores de TH-67

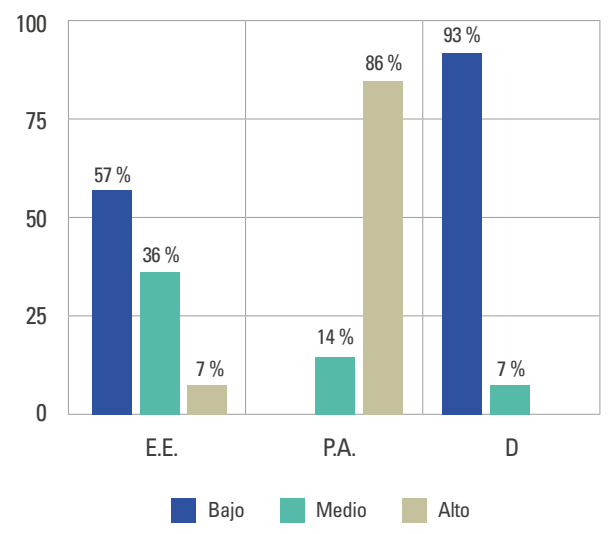

Figura 1. Análisis comparativo, tres dimensiones en instructores de TH-67 Fuente: Arboleda (2019).
Las condiciones laborales de los instructores de TH-67 obtenidas por medio del cuestionario y analizadas estadísticamente a través de medidas de tendencia central, se asocian tanto a las operaciones de instrucción y vuelo, como a las labores administrativas. Los datos fueron analizados estadísticamente a través de medidas de tendencia central, entre las cuales se encuentran algunas mediciones de posición como media y moda (frecuencia), y medidas de dispersión como la desviación estándar, que permitió analizar de una manera más detallada la clasificación de las variables y datos obtenidos en la población objeto de estudio (Hall, 2006).

Si bien la muestra inicial del análisis fue de 27 instructores, por cuestiones diversas, algunos fueron desertando la investigación, de modo que, se obtuvo una muestra final de 14 participantes, con una edad promedio de $35 \pm 1,95$ años; con edad mínima de 32 años y máxima de 39 años, todos de sexo masculino. El $71,43 \%$ (n: 10) casados y el $28,57 \%$ (n: 4) solteros.

Dentro del análisis de las condiciones laborales, a la pregunta: ¿cuántos años lleva ejerciendo como piloto?, se evidenció que 6 instructores laboran como pilotos de la FAc hace 1 a 4 años, es decir el 42,86 \% de la población; 1 instructor de 5 a 8 años que equivale al 7,14 \%; 2 instructores de 9 a 12 años que equivalen al $14,29 \%$; 4 instructores de 13 a 16 años que equivalen al 28,57\%, y 1 instructor de 17 a 20 años que equivale al $7,14 \%$. Lo anterior equivale a que, como instructores de TH-67 el 42,86 \% (n: 6) lleva laborando menos de un año, el 42,86\% (n: 6) lleva entre 1 y 4 años, y el 14,29\% (n: 2) lleva 5 o más años.

De igual manera, se encontró que el 78,57 \% ( $n$ : 11) inició con autonomía como instructor en el equipo TH-67, y el 21,43\% (n: 3 ) tenía experiencia como instructor en otra aeronave (equipo). También se observó que el 42,86 \% (n: 6) de los instructores no tiene cargo administrativo, y el 57,14\% (n: 8) sí lo tiene. De los oficiales con cargo administrativo, el 57,14\% (n: 8) lleva de 1 a 2 años de antigüedad en el cargo y el 7,14 \% (n: 1) labora hace más de 4 años en el cargo.

En relación con los instructores de cargo administrativo, el 28,57 \% (n: 4) dedica 0 horas al cargo administrativo, el 21,43\% (n: 3 ) dedica de 1 a 3 horas al 
cargo, y el 50 \% (n: 7) labora de 4 a 6 horas en el cargo. En términos de las actividades relacionadas con instrucción, se observa que el 100 \% (n: 14) de la población dedica entre 7 y 9 horas al día a labores relacionadas este factor. Se establece que el 85,71\% (n: 12) dedica de 1 a 3 horas al día para preparar sus clases y el 14,29\% (n: 2) de los pilotos instructores no prepara clase. En cuanto al periodo de vuelo (fase), el 35,71\% $(n: 5)$ se encuentra en contacto con el lugar de vuelo, el 7,14 \% (n: 1) en táctico, el 42,86\%, (n: 6) en la fase que se vuela con lentes de visión nocturna (NVG), y el $14,29 \%$ (n: 2) en instrumentos. En cuanto a horas de vuelo, el promedio de horas voladas en el equipo es de $797 \pm 677$, y el promedio de horas grupal en general como pilotos instructores, es de $676 \pm 545$. Respecto a la reasignación de alumnos a los instructores, se puede decir que el $28,57 \%$ (n: 4) no tiene, y el 71,43\% (n: 10) sí tiene reasignación. El 35,71\% (n: 5) de los instructores no ha tenido cambios en la programación de instrucción, y el 64,29 \% (n: 9) sí ha tenido cambios. Por último, se observó que el $100 \%$ de la población de instructores tiene descanso de vuelo los fines de semana (tabla 2).

Tabla 2.

Caracterización de las condiciones laborales de los instructores de TH-67

\begin{tabular}{|l|l|c|}
\hline Edad promedio & 35 años & \\
\hline Sexo & Masculino & \\
\hline \multirow{2}{*}{ Estado civil } & Soltero & $28,6 \%$ \\
\hline \multirow{2}{*}{ Años como instructor de TH-67 } & Casado & $71,4 \%$ \\
\hline \multirow{2}{*}{ Instructores con carga administrativa } & Menos 1 año & $42,9 \%$ \\
\hline & 1 a 4 años & $42,9 \%$ \\
\cline { 2 - 3 } & 5 años o más & $14,2 \%$ \\
\hline Horas al día dedicadas a la instrucción & No & $57,1 \%$ \\
\hline Promedio de horas como instructor & 676 & $42,9 \%$ \\
\hline Descanso de vuelo el fin de semana & Sí & $100 \%$ \\
\hline Fuente: Arboleda (2019). & \multicolumn{2}{|l}{} \\
\hline
\end{tabular}

Para establecer posibles asociaciones entre las diferentes dimensiones del test y los datos obtenidos en carga administrativa y horas de instrucción, los resultados se presentan en la siguiente tabla (tabla 3 ):
Tabla 3.

Resultados del análisis exploratorio de chi cuadrado

\begin{tabular}{|c|c|c|c|c|}
\hline Variables & $\begin{array}{l}\text { Grados de } \\
\text { libertad }\end{array}$ & $\mathbf{P}$ & Chi & Valor \\
\hline $\begin{array}{l}\text { Horas de preparación } \\
{ }^{*} \text { Agotamiento emocional }\end{array}$ & 2 & 0,122 & 5,991 & 4,200 \\
\hline $\begin{array}{l}\text { Horas de preparación } \\
{ }^{*} \text { Realización personal }\end{array}$ & 1 & 0,119 & 3,841 & 2,431 \\
\hline $\begin{array}{l}\text { Horas de preparación } \\
\text { * Despersonalización }\end{array}$ & 1 & 0,672 & 3,841 & 0,179 \\
\hline $\begin{array}{l}\text { Tiempo en el cargo } \\
\text { administrativo } \\
{ }^{*} \text { Agotamiento emocional }\end{array}$ & 4 & 0,520 & 9,488 & 3,229 \\
\hline $\begin{array}{l}\text { Tiempo en el cargo } \\
\text { administrativo } \\
{ }^{*} \text { Realización personal }\end{array}$ & 2 & 0,417 & 5,991 & 1,750 \\
\hline $\begin{array}{l}\text { Tiempo en el cargo } \\
\text { administrativo } \\
\text { * Despersonalización }\end{array}$ & 2 & 0,379 & 5,991 & 1,938 \\
\hline $\begin{array}{l}\text { Horas en cargo adicional } \\
{ }^{*} \text { Agotamiento emocional }\end{array}$ & 4 & 0,225 & 9,488 & 5,667 \\
\hline $\begin{array}{l}\text { Horas en cargo adicional } \\
{ }^{*} \text { Realización personal }\end{array}$ & 2 & 0,311 & 5,991 & 2,333 \\
\hline $\begin{array}{l}\text { Horas en cargo adicional } \\
\text { * Despersonalización }\end{array}$ & 2 & 0,260 & 5,991 & 2,692 \\
\hline $\begin{array}{l}\text { Carga administrativa } \\
{ }^{*} \text { Agotamiento emocional }\end{array}$ & 2 & 0,627 & 5,991 & 0,933 \\
\hline $\begin{array}{l}\text { Carga administrativa } \\
{ }^{*} \text { Realización personal }\end{array}$ & 1 & 0,186 & 3,841 & 1,750 \\
\hline $\begin{array}{l}\text { Carga administrativa } \\
\text { * Despersonalización }\end{array}$ & 1 & 0,231 & 3,841 & 1,436 \\
\hline $\begin{array}{l}\text { Años de instructor en TH-67 } \\
{ }^{*} \text { Agotamiento emocional }\end{array}$ & 4 & 0,113 & 9,488 & 7,467 \\
\hline $\begin{array}{l}\text { Años de instructor en TH-67 } \\
\text { * Realización personal }\end{array}$ & 2 & 0,823 & 5,991 & 0,389 \\
\hline $\begin{array}{l}\text { Años de instructor en TH-67 } \\
\text { * Despersonalización }\end{array}$ & 2 & 0,488 & 5,991 & 1,436 \\
\hline
\end{tabular}

Fuente: Arboleda (2019).

\section{Discusión de resultados}

A partir de los resultados obtenidos, se observa que el síndrome de burnout se presenta en bajo porcentaje (P) en la población de instructores de la Escuela de Helicópteros de la Fuerza Pública. Al realizar el análisis de la población objeto de estudio, en las tres dimensiones, los resultados indicaron que el ítem agotamiento emocional (A.E) está en un $57 \%$ con nivel bajo, seguido del nivel medio con $36 \%$, y el $7 \%$ de la población 
presenta un nivel alto de agotamiento emocional. Por otro lado, en el ítem realización personal (P.A) se puede evidenciar que no existe población en el ítem bajo, $y$, al contrario, la población se encuentra en los parámetros medio y alto, siendo el $14 \%$ de nivel medio y $86 \%$ de nivel alto, lo que indica que gran porcentaje de la población se encuentra satisfecha con el trabajo que realiza. Por último, al analizar el ítem de despersonalización (D) se puede decir que el 93 \% de la población objeto de estudio se encuentra en el nivel bajo, el restante $7 \%$ se encuentra en nivel medio, y no existe población en nivel alto de despersonalización.

Al evaluar la influencia de los factores laborales, no se evidenció una relación significativa de estos con las dimensiones del test de Maslach. Sin embargo, se observó que la despersonalización y el agotamiento emocional son bajos en la población estudiada, mientras que los puntajes de realización personal están en los niveles medio y alto. Este hallazgo sugiere que en la población objeto de estudio, la dimensión de realización personal puede estar influenciada por los años como instructor en el equipo, que en promedio de horas para la población es de $676 \pm 545$, lo que finalmente se deriva en un mayor reconocimiento por la institución dada la experiencia en vuelo. Los resultados de esta investigación son contrarios a lo reportado en los estudios de docentes universitarios de algunos países latinoamericanos, en los cuales según Botero-Álvarez (2012), se observó que la población fue afectada por factores como la multiplicidad de tareas y la falta de reconocimiento. Si bien algunas de estas causas están presentes en la Escuela de Helicópteros, y por tanto, afecta a sus instructores, gracias al ambiente en el que desarrollan su experiencia, se posicionan en lugares reconocidos y destacados por todo el personal de pilotos e integrantes de la Escuela, lo que a la vez, se convierte en un motivador importante y favorece la alta realización personal de la población objeto de estudio.

La realización personal también se relaciona con los años que los instructores llevan realizando sus actividades como pilotos, esta experiencia asociada a la invitación como instructores de otras fuerzas, hace que los pilotos que ingresan a la Escuela de Helicópteros sean personal con experiencia en vuelo de entre
1.000 y 2.000 horas totales, equivalentes al grado de Capitán a Mayor. Lo anterior, les permite desempeñarse de forma íntegra ante las exigencias de la formación de vuelo.

Ahora bien, los resultados bajos de despersonalización y agotamiento emocional pueden ser explicados debido a diferentes estímulos recibidos por los instructores a nivel militar, y por el reconocimiento como instructores por parte de comandantes y alumnos. Así mismo, se observa que pese a las horas de instrucción, la población objeto de estudio tiene horas de descanso los fines de semana, lo que les permite tener tiempo libre para realizar otras actividades diferentes a la instrucción. En la población estudiada no se evidenció que la carga administrativa estuviera asociada al agotamiento emocional, el cual tuvo bajo nivel en la mayoría de la población, pero un 36 \% lo presentó en un nivel medio. Es importante recordar que no todos los instructores presentan carga administrativa, de la población estudiada, solamente el 57,14\% presenta un cargo administrativo.

De los oficiales que tienen labores administrativas, el 57,14 \% lleva de 1 a 2 años de antigüedad en el cargo, y el 7,14 \% labora hace más de 4 años en este cargo. Para entender la condición laboral de la población, se específica que el cargo administrativo es aquel en el cual el oficial debe desempeñarse como comandante de un grupo, sección o dependencia, lo que implica responsabilidades adicionales al tener que administrar personal y además, hacer formaciones militares, elaboración de contratos, revisión de documentos, entrenamiento físico y responsabilidades gerenciales como la toma de decisiones, que tienen gran impacto en los procesos de la organización.

Por otra parte, de acuerdo con los convenios establecidos dentro de las Fuerzas Armadas, se encuentra el intercambio de pilotos instructores del Ejército y Armada para que se desempeñen como instructores. Los oficiales del Ejército no son trasladados de planta, motivo por el cual no les asignan cargos administrativos y solo desempeñan labores relacionadas a la instrucción. Por el contrario, los instructores de la Armada son trasladados como oficiales de planta, por consiguiente, les asignan cargos operativos dentro de 
la estructura de la Escuela. A su vez, al interior de la población de instructores militares se encuentran los oficiales invitados de la FAC, quienes también desempeñan cargos administrativos adicionales a los relacionados directa e indirectamente con la instrucción. Para los instructores invitados, el cargo administrativo puede representar las responsabilidades de organización documental y programación de instructores de vuelo. Lo anterior evidencia que la carga laboral es significativamente diferente para los instructores invitados y los de planta, pudiendo ser este factor un causante de estrés o cansancio para los instructores. Aunque en el estudio no se encontró una asociación significativa de la carga administrativa con las dimensiones del test Maslach, es posible que este pueda convertirse en un factor asociado al síndrome de burnout, por tanto, debe ser tenido en cuenta como complemento en el estudio del síndrome en otros campos.

En relación con las horas para la preparación de clases, aunque no hubo una asociación estadística significativa con las dimensiones del test Maslach, se observó que el 85,71 \% de pilotos instructores dedica al menos de 1 a 3 horas para preparar sus clases del día siguiente, y el 14,29\% de los pilotos instructores no lo hace. Este resultado mostró que el mismo porcentaje de pilotos que no prepara sus clases (14,29\%), se corresponde con el tiempo de experiencia del instructor (5 años). Es decir que, la diferencia en horas dedicadas a la preparación de clases está relacionada con la experiencia y años dedicados a la instrucción, siendo menor el tiempo para los instructores con mayor experiencia.

Dentro de las horas al día de instrucción, se cuentan todas las labores realizadas antes, durante y después del vuelo: briefing de instructores, entiéndase briefing como el repaso de maniobras, rutinas y conocimientos académicos necesarios para desarrollar un vuelo seguro, tiene una duración aproximada de $45 \mathrm{mi}$ nutos; briefing de alumnos, con duración aproximada de 45 minutos; prevuelo, constituido por las acciones de revisión y verificación de las condiciones técnicas de las aeronaves para el cumplimiento de la misión de entrenamiento, que tiene una duración aproximada de 30 minutos; vuelo de instrucción con duración de 3 horas; postvuelo, conformado por las acciones de revisión y verificación de las condiciones técnicas de las aeronaves al finalizar el vuelo, con duración aproximada de 30 minutos; post briefing con el alumno, en el que se explican y retroalimentan las maniobras efectuadas en el vuelo de instrucción, tiene una duración aproximada de 30 minutos; calificación de alumnos, la cual tiene una duración aproximada de 30 minutos. El periodo de vuelo (fase) en el que está el instructor, representa la exposición a diferentes ambientes laborales. En la fase de contacto estaban volando 5 pilotos que representan el $35,71 \%$, en la fase de táctico 1 piloto que representa el $7,14 \%$, en la fase que se vuela con lentes de visión nocturna (NVG) 6 pilotos que representan el $42,86 \%$, y en la fase de simulador de vuelo (instrumentos) 2 pilotos que representan el $14,29 \%$.

Respecto a los datos anteriores, cabe resaltar que los pilotos que se encuentran en las fases de contacto y táctico, están expuestos a características de la operación de las aeronaves de ala rotatoria como son fuerzas gravitacionales, peligros tóxicos, temperaturas extremas, ruido y vibración, además de la alta densidad de tránsito aéreo del aeródromo. De igual manera, los instructores que se encuentran en la fase NVG están expuestos a las condiciones de contacto y táctico, mientras operan con lentes de visión nocturna que agregan limitaciones a los pilotos, por ejemplo, cambio de la percepción de profundidad y disminución de la agudeza visual 20-20.

Vale la pena destacar que las horas voladas en la aeronave representan la experiencia operativa del instructor con el equipo, muestra la confianza y la destreza con la que se puede desempeñar. Por ese motivo, la Escuela de Helicópteros toma esta como un insumo esencial para programar al piloto en las diferentes fases de vuelo. De acuerdo con los estándares de la Escuela de Helicópteros, un instructor que tenga más de 200 horas está capacitado para volar en cualquier fase. Los pilotos que se desempeñan como instructores en la Escuela, normalmente no realizan vuelos que no sean de instrucción o que tengan que ver con mantenimiento del orden público (operativos), debido a que les representaría perder el tiempo de descanso y libre esparcimiento que tienen después de exponerse a las 
largas jornadas durante la semana. De acuerdo con la jerarquía de necesidades de Maslow (Chiavenato, 2007), el comportamiento del ser humano depende de cómo supla sus necesidades básicas y la autorrealización es una de las necesidades básicas más elevadas. Lograr utilizar todo su potencial para desarrollarse en el ambiente en el que la persona se encuentra, es un motivador muy importante que hace que el instructor de vuelo siempre esté luchando por alcanzar más metas y objetivos personales.

Al poseer el deseo de autorrealización, se podrán motivar otros aspectos de la vida que representan bienestar y mitigación de elementos negativos. Estos motivadores son la independencia, la autonomía, el estatus, la utilización plena de sus talentos y el reconocimiento social. En ese sentido, los altos niveles de realización personal pueden mitigar el agotamiento emocional y la despersonalización en la población estudiada, al contrario de lo que afirman los estudios reportados en personal docente.

En el test Maslach, el agotamiento emocional se mide con nueve preguntas a través de las cuales se valoran las actitudes, sentimientos negativos o cínicos, acerca de los clientes o usuarios, traducidos en una deshumanizada percepción de los demás, partiendo de la pregunta ¿Al final de la jornada me siento agotado? Si bien en el Código Sustantivo del Trabajo en Colombia se deben cumplir máximo 8 horas diarias de trabajo, las condiciones laborales de los militares son diferentes. La Aeronáutica Civil establece los tiempos de descanso que deben cumplir las tripulaciones luego de haber realizado sus funciones de vuelo, de acuerdo con los Reglamentos Aeronáuticos de Colombia. Al realizar las preguntas 7,8 y 9 sobre condiciones laborales, se pudo evidenciar que las jornadas laborales en algunos individuos en la EHFAA se extienden de 11 a 18 horas de trabajo diario, superando en rangos bastantes altos lo estipulado, lo que puede contribuir a que se presente agotamiento emocional. No obstante, debe tenerse en cuenta que la profesión de militar y pilotaje, ocupan el primer y el tercer lugar, respectivamente, en el ranking de empleos que soportan más presión según estudios realizados por la agencia estadounidense Career Cast (Chiavenato, 2007).
En consecuencia, para algunas áreas se considera que el instrumento de Maslach no es exacto al determinar la presencia del burnout, es necesario crear instrumentos que contemplen a los alumnos o a los compañeros de trabajo, y no solo la subjetividad de la respuesta dada por los instructores.

\section{Conclusiones}

La presencia del síndrome de burnout en los pilotos instructores de TH-67 de la Escuela de Helicópteros de las Fuerzas Armadas, no es frecuente. Esto debido a que en la dimensión de agotamiento emocional la mayoría de la población presentó un nivel bajo con $57,14 \%$, y solo un $7 \%$ reportó nivel alto de agotamiento. Por cuanto a la dimensión de realización personal, el nivel fue alto ocupando el 85,71 \% y en la dimensión de despersonalización, el nivel fue bajo con el $92,86 \%$ de los pilotos instructores evaluados. Así mismo, no se observó asociación entre los factores laborales (horas de preparación, tiempo en el cargo administrativo, horas en cargo adicional, carga administrativa y años de instructor en $\mathrm{TH}-67$ ).

Estos resultados difieren con los reportados en la literatura por docentes universitarios, pese a la carga en términos de intensidad, frecuencia, sobrecarga y responsabilidad laboral reportada de los instructores. Al caracterizar las condiciones laborales de la población de estudio, se pudo observar que los pilotos instructores desarrollan actividades relacionadas directamente con la instrucción y el vuelo de instrucción, entre las cuales están: realización de actividades académicas conformadas por preparación de los temas, revisión de diapositivas y programación de didácticas para el desarrollo de las clases, y demás actividades previas. A la vez, los instructores deben dar clase según el tema y la fase de vuelo en que se encuentren (contacto, táctico, instrumentos y visores) o a la cual sean reasignados, y deben realizar evaluación de las actividades académicas tanto prácticas como teóricas. Además de los trabajos académicos, los instructores deben realizar actividades relacionadas directamente 
con la instrucción de vuelo; por ejemplo, el briefing con los instructores (verificación de temas relacionados con la seguridad y maniobras a realizar en la misión), briefing con los alumnos (socialización y control de las maniobras a realizar en el vuelo de instrucción con los alumnos), para posteriormente realizar pre-vuelo (verificación de que la aeronave se encuentre en condiciones óptimas para realizar un vuelo seguro), el cual se hace de manera conjunta con el alumno, en el que se verifica el grado de conocimiento de los sistemas que se van a operar o pueden fallar durante la misión. Posterior a las actividades mencionadas, los instructores realizan el vuelo, que puede ser real o en simulador. De acuerdo con el plan de instrucción y entrenamiento enseñan todas la maniobras vistas en el briefing que serán evaluadas al final de la misión, luego se realiza el post-briefing (retroalimentación de las fortalezas y debilidades encontradas durante la misión) y al final, los instructores deben realizar la calificación de vuelo (de acuerdo al formato de calificación de la EHFAA).

Según los hallazgos realizados en la caracterización de la condición laboral, se puede observar que el 42,86 \% de la población tiene un tiempo de servicio como instructor en la Escuela menor a un año. El $57,14 \%$ (n: 8), perteneciente al cargo administrativo lleva de 1 a 2 años de antigüedad en el cargo. La Escuela de Helicópteros tiene instructores del Ejército Nacional, la Armada, la Fuerza Aérea y extranjeros invitados de otras fuerzas, por lo tanto, a algunos de ellos (dependiendo el convenio), tienen o no asignado un cargo administrativo dentro de los cuales están los pilotos de la FAC, la Armada y extranjeros. Frente a estos datos, el 57,14 \% de la población tiene cargo administrativo y el $42,86 \%$ no lo tiene; de los que realizan labores administrativas, el 28,57\% dedica menos de 1 hora al día, el 21,43\% dedica entre 1 a 3 horas al día, y el $50 \%$ dedica entre 4 a 6 horas al día a labores administrativas adicionales a las labores de instrucción y vuelo. Así mismo, se pudo evidenciar que es frecuente la reasignación de horario y de alumnos, lo que sugiere exigencia extra y posible estrés en los instructores que deben ajustar sus clases, horarios y redistribuir las tareas diarias, convirtiéndose esta en una característica laboral que podría favorecer la aparición del burnout.
Al utilizar el test de Maslach Inventory como herramienta para determinar la presencia del síndrome de burnout en los instructores de TH-67, se puede concluir que la aplicación de esta herramienta tiene limitaciones para identificar de manera completa la condición de los instructores en los ítems de realización personal y despersonalización, debido a que es evaluada de forma subjetiva. Para complementar este tipo de evaluación, se considera que la despersonalización debe ser revisada de manera simultánea por los alumnos que reafirmen el trato recibido por los instructores, hallazgo que se ha presentado en estudios previos, ya que en la evaluación que se da, el instructor puede variar al pensar que su trato es adecuado y cordial. De igual manera, se analiza que la condición y estatus del instructor dentro de la organización puede interferir en la autocrítica, lo que podría convertirse en un sesgo en los resultados.

Al hacer un análisis de los factores laborales de los instructores de TH-67 que más afectan la prevalencia y afectación de cada uno de los ítems analizados del burnout, no se puede concluir con seguridad si la carga administrativa afecta el agotamiento emocional que en la población estudiada fue bajo. Sin embargo, esta carga a mayor tiempo e intensidad puede causar agotamiento emocional, que para Patlán (2013) se presenta de manera progresiva y podría desencadenar en el individuo pérdida paulatina de energía, interés y disposición para realizar labores asignadas, debido al cansancio, fatiga y desgaste que producen las tareas inherentes al cargo que desarrollan, o en el caso de los instructores de la FAC, las largas jornadas a las que se encuentran expuestos con labores adicionales como formaciones militares, elaboración de contratos, revisión de documentos, entrenamiento físico y responsabilidades gerenciales en la toma de decisiones.

El cargo administrativo para instructores invitados, podría verse permeado por factores estresores o de cansancio emocional debido a las tareas significativamente diferentes con que tienen con los instructores de planta, como la organización documental y programación de instructores de vuelo.

La despersonalización de los instructores, aunque se encontró baja, podría verse influenciada por las 
horas que los instructores dedican a preparar clases, lo cual depende directamente de la experiencia y años como instructor, a mayor experiencia y años, dedican menos tiempo a la preparación de clases. Además, la realización personal puede verse afectada por los años que llevan desempeñándose como instructores, a mayor tiempo es mayor la realización personal, en el caso de la población objeto de estudio el $86 \%$ experimenta una realización alta.

\section{Referencias bibliográficas}

Alonso, M. (2009, 11 de junio). Actividad aeroespacial, seguridad y salud mental [ponencia]. IV Congreso Argentino de Salud Mental. Buenos Aires, Argentina. https://www. modestoalonso.com.ar/assets/7_actividad_aeroespa cial_seguridad_y_salud_mental_(2009).pdf

Arboleda, M. A. (2019). Estudio de presencia y afectación del síndrome de burnout en instructores de TH-67, en la escuela de helicópteros de las Fuerzas Armadas de Colombia [Tesis de maestría]. Escuela de Posgrados de la Fuerza Aérea Colombiana. Repositorio de Biblioteca.

Bernal, C. A. (2010). Metodología de la investigación (3ra ed.). Pearson Educación de Colombia.

Botero-Álvarez, C. (2012). Riesgo psicosocial intralaboral y "burnout" en docentes universitarios de algunos países latinoamericanos. Journal of Social Issues, 30(1), 159-165.

Chiavenato, I. (2007). Administración de recursos humanos. El capital humano de las organizaciones. McGraw-Hill. https://cucjonline.com/biblioteca/files/original/aec4d of8da9f45c14d9687966f292cd2.pdf

Estrella-Silva, M. G., \& Portalanza, A. (2015). El burnout y su aplicación en el sector de la aviación: una aproximación conceptual. Suma de Negocios, 6(14), 204-211. http://dx. doi.org/10.1016/j.sumneg.2015.10.004

Fuerza Aérea Colombiana [FAC]. (2016). Estadísticas de factor humano y operacional 2014-2016. [Documento interno FAC].

Golembiewski, R. T., Munzenrider, R., \& Carter, D. (1983). Phases of Progressive Burnout and Their Work Site Covariants: Critical Issues in OD Research and Praxis. The Journal of applied behavioral science, 19(4), 461-481. https://doi. org/10.1177/002188638301900408

Hall, P. (2006). Medidas de tendencia central. Oficina Nacional del Registro Civil. https://www.onsc.gub.uy/enap/
images/stories/MATERIAL_DE_CURSOS/Clase_V_Medidas_de_tendencia_central.pdf

Hernández-Sampieri, R., Fernández-Collado, C., \& Baptista-Lucio, M. (2014). Metodología de la investigación (6ta ed.). McGraw-Hill.

Martínez, J. P., Méndez, I., Secanilla, E., \& Gónzalez, E. (2012). Evolución de los niveles de burnout en un estudio comparativo en cuidadores profesionales tras una situación de estrés postraumático. EJIHPE: European Journal of Investigation in Health, Psychology and Education, 2(1), 2939. https://doi.org/10.30552/ejihpe.v2i1.25

Maslach, C., Jackson, S. E., Leiter, M. P., Schaufeli, W. B., \& Schwab, R. L. (1986). Maslach burnout inventory. Consulting psychologists press (21), 3463-3464. https://doi. org/10.12691/ajnr-7-5-7

Montoya-Zuluaga, P., \& Moreno-Moreno, S. (2012). Relación entre síndrome de burnout, estrategias de afrontamiento y engagement. Psicología desde el Caribe, 29(1), 205227. https://www.redalyc.org/pdf/213/21323171011.pdf

Olivares-Faúndez, V., \& Gil-Monte, P. (2009). Análisis de las principales fortalezas y debilidades del "Maslach Burnout Inventory” (MBI). Ciencia \& Trabajo, 11, 160-167. https://n9.cl/2k4a

Pando-Moreno, M., Aranda-Beltrán, C., \& López-Palomar, M. D. (2015). Validez factorial del Maslach Burnout Inventory-General Survey en ocho países latinoamericanos. Ciencia \& trabajo, 17(52), 28-31. https://doi.org/10.4067/ S0718-24492015000100006

Pérez, J. P. (2013). Efecto del burnout y la sobrecarga en la calidad de vida en el trabajo. Estudios gerenciales, 29(129), 445-455. https://www.sciencedirect.com/science/arti cle/pii/S0123592314000813

Quiceno, J. M., \& Vinaccia-Alpi, S. (2007). Burnout: "síndrome de quemarse en el trabajo (SQT)". Acta Colombiana de Psicología, 10(2), 117-125. https://www.redalyc.org/ pdf/798/79810212.pdf

Rholetter, W. (2013). Burnout (psychology). Salem Press encyclopedia.

Rubio, L. M. S. (2010). El estudio del factor humano en accidentes de aviación. Pensamiento Psicológico, 7(14). https://revistas.javerianacali.edu.co/index.php/pensa mientopsicologico/article/view/13

Sierra-Siegert, M. (2008). La despersonalización: aspectos clínicos y neurobiológicos. Revista Colombiana de Psiquiatría, 17(1), 40-55. http://psiquiatria.org.co/web/wp-content/ uploads/2012/04/VOL-37/1/37-1.pdf\#page=40 